\title{
Weight and skeletal maturation - a study of radiological and chronological age in an anorexia nervosa population
}

\author{
J. H. LACEY* \\ M.B., M.Phil., M.R.C.Psych. \\ A. H. CRISP $\dagger$ \\ M.D., F.R.C.P., F.R.C.P.Ed., F.R.C.Psych., D.P.M. \\ G. HART $\dagger$ \\ M.B. B.S., F.F.R. \\ B. A. KIRKWOOD + \\ B.Sc.
}
*Academic Department of Psychiatry, Middlesex Hospital Medical School; †St George's Hospital Medical School; St George’s Hospital and $\ddagger$ Chelsea College

\begin{abstract}
Summary
The carpal bones of 18 anorexia nervosa patients were radiographed and the $X$-ray age assessed by 2 different standard methods

The study demonstrates that skeletal development in anorexia nervosa patients is delayed such that there is no association between radiological assessment of age and chronological age. It is strongly suggested that bony development actually ceases when body weight falls sufficiently to stop menstruation. There was a highly significant $(P=0.001)$ linear relationship between radiological age and the sum of the age of onset of the illness plus any period(s) of re-feeding. Weight gain seems to re-kindle the bone maturing mechanisms: the role of weight thresholds and associated hormone activity being discussed.

The findings of this study strongly support the existing evidence that the anorexia nervosa patient is biologically and psychologically immature.
\end{abstract}

\section{Introduction}

It has been claimed that there are critical weight thresholds which relate growth to reproductive potential. There are suggestions of 2 such thresholds in pubescent girls. The first and lower one has been determined in anorectic female patients. In them, pituitary responsiveness to luteinizing hormone-releasing hormone (LHRH) only occurred at weights above $44 \mathrm{~kg}$ (Palmer et al., 1974). This threshold seems to generate initial femininity both psychologically and biologically. The second and higher threshold heralds menstruation. Frisch and her colleagues (Frisch and McArthur, 1974) have

Requests for reprints: Dr J. H. Lacey, Senior Lecturer and Honorary Consultant, Academic Department of Psychiatry, Middlesex Hospital Medical School, London W.1. suggested that menarche becomes possible when the body comprises at least $17 \%$ fatty tissue and has reached a mean of $48 \pm 5 \mathrm{~kg}$. They suggest that this critical weight and fat tissue volume trigger basal metabolic changes which in turn initiate sexual changes.

Anorexia nervosa has been construed as a phobia of normal adolescent weight, the origins of which are pubertal. It has been claimed that it reflects a need to avoid maturity, low body weight being achieved by major carbohydrate abstention or by bulimia and induced vomiting (Crisp, 1970).

In an 'abstaining' anorectic population Kalucy (Kalucy et al., 1976) has shown lack of surges of growth hormone during the first $2 \mathrm{hr}$ of sleep. Crisp (1969) reported that female patients whose anorexia had begun early in adolescence tended to be shorter in stature than a control population matched for age and social class. More recently a detailed case study of one male anorectic showed stunting of growth and delayed puberty and maturation in terms of skeletal age (Toms and Crisp, 1972). Work (Prader, Tanner and von Harnack, 1963) with a younger age group of both sexes has shown that starvation from a number of causes will lead to stunted growth; this work also demonstrated the phenomenon of growth 'catch-up' when normal nutrition recommenced. Similarly Money (1975) and other workers, e.g. Powell, Hopwood and Barratt (1973) working with 'psychosocial dwarfs', have reported how these malnourished stunted patients, when re-fed, have higher plasma growth hormone levels and grow.

This evidence has led the present authors to believe that the bones of chronic anorectics who weigh $\leqslant 43$ $\mathrm{kg}$ might remain in a 'prepubertal state' mirroring 
their hormonal and psychological pictures. Also they thought it probable that re-feeding through the critical thresholds would allow the bones to mature and tend to 'catch up' with their chronological age in a way similar to that witnessed in hormonal and psychological studies. This study was undertaken to examine this hypothesis.

\section{Methods}

The study was conducted on 18 anorexia nervosa patients, 3 of whom were male. The criteria used in making the diagnosis were those described by Crisp (1970). Since the aim of the study was to determine the effect of anorexia nervosa on bone maturation no patient who had become ill after the age of 18 years was included because, beyond this age, bones cease to be a helpful indicator of development. Also no anorectic who had induced vomiting, or purged him/herself was included since either of these actions may cause gross electrolyte abnormalities. None of the patients taking part had received any artificial hormonal treatment including the contraceptive pill. The purpose of the study was explained to the patients and their informed consent was given.

After extensive interviews with the patients and their parents, and an examination of the medical notes, an assessment was made of the age of onset of the illness, as defined by the cessation of menstruation or, if menstruation had never begun, the occurrence of major weight loss together with a clear pathological pursuit of thinness and a weight phobia (Crisp, 1970) An assessment was also made of the amount of time, since the onset of the illness, during which the patient was eating adequately. This was judged to be occurring when the patient's weight was increasing regularly, whether in hospital or at home, over a sustained period of at least 2 months, or when his/her weight was stable and within $10 \%$ of a target weight, based on his/her present height and age at which anorexia nervosa had begun.

All but 4 of the patients had been admitted to hospital for periods of time when they had been given a normal diet containing normal amounts of carbohydrates in order to reach their target weights. They had thus been re-fed to the point, in terms of body weight and fatty volume, when their anorexia supervened. No special food or supplement was given and no psychotropic medication prescribed within at least the 6 months' period before the study.

The hands and wrists of the patients were radiographed to show bony and epiphyseal shapes. Most examinations were of good quality without repetition, so that radiation was almost minimal. The patients were coded and the only information recorded on the radiographs was the patient's code number and sex.

The radiographs were then assessed for bone age by comparison with standard radiographs published by Greulich and Pyle (1959). By this method (G-P) radiological ages up to 18 years can be predicted.

Some months later these radiographs were reassessed. The radiological age was evaluated by awarding a score to the radius, ulna and small bones as described by Tanner, Whitehouse and Marshalk (1975). This method (TW2[RUS]) allows deduction of radiological ages up to 16 years for females and 18 years for males.

\section{Results}

The sex and age of the 18 patients together with the age of onset of anorexia nervosa and the amount of time spent re-feeding are shown in Table 1. Also shown are their radiological ages as assessed by the 2 methods G-P and TW2(RUS). The data were

TABle 1. Data (in years) for 18 patients with anorexia nervosa

\begin{tabular}{|c|c|c|c|c|c|c|c|}
\hline \multirow[b]{2}{*}{ Subject } & \multirow[b]{2}{*}{ Sex } & \multirow{2}{*}{$\begin{array}{c}\text { Chronological } \\
\text { age }\end{array}$} & \multirow{2}{*}{$\begin{array}{l}\text { Age of onset } \\
\text { (1) }\end{array}$} & \multirow{2}{*}{$\begin{array}{l}\text { Period of } \\
\text { re-feeding } \\
\text { (2) }\end{array}$} & \multirow{2}{*}{$\begin{array}{r}\text { Total } \\
(1)+(2)\end{array}$} & \multicolumn{2}{|c|}{ Radiological age } \\
\hline & & & & & & G-P & TW2(RUS) \\
\hline 1 & $\mathbf{F}$ & $17 \cdot 1$ & $13 \cdot 5$ & $2 \cdot 0$ & $15 \cdot 5$ & $16 \cdot 0$ & $15 \cdot 3$ \\
\hline 2 & $\mathbf{F}$ & $20 \cdot 3$ & $15 \cdot 5$ & $1 \cdot 2$ & $16 \cdot 7$ & $17 \cdot 0$ & 16.0 \\
\hline 3 & $\mathbf{F}$ & $20 \cdot 1$ & $16 \cdot 3$ & 0.0 & $16 \cdot 3$ & $16 \cdot 0$ & $15 \cdot 5$ \\
\hline 4 & $\mathbf{F}$ & $18 \cdot 5$ & $15 \cdot 3$ & 1.9 & $17 \cdot 2$ & $18 \cdot 0$ & $16 \cdot 0$ \\
\hline 5 & $\mathbf{F}$ & $16 \cdot 8$ & $14 \cdot 2$ & 0.0 & $14 \cdot 2$ & $14 \cdot 0$ & $15 \cdot 0$ \\
\hline 6 & $\mathbf{F}$ & $21 \cdot 3$ & $17 \cdot 0$ & $1 \cdot 3$ & $18 \cdot 3$ & $18 \cdot 0$ & $16 \cdot 0$ \\
\hline 7 & $\mathbf{F}$ & $14 \cdot 7$ & $14 \cdot 2$ & 0.0 & $14 \cdot 2$ & $13 \cdot 0$ & $13 \cdot 1$ \\
\hline 8 & $\mathbf{F}$ & $19 \cdot 5$ & $12 \cdot 0$ & 0.0 & $12 \cdot 0$ & $12 \cdot 0$ & $12 \cdot 1$ \\
\hline 9 & $\mathbf{M}$ & $17 \cdot 8$ & $13 \cdot 8$ & $3 \cdot 5$ & $17 \cdot 1$ & $18 \cdot 0$ & $17 \cdot 1$ \\
\hline 10 & $\mathbf{M}$ & $21 \cdot 2$ & $14 \cdot 0$ & $1 \cdot 2$ & $15 \cdot 2$ & $15 \cdot 5$ & $15 \cdot 9$ \\
\hline 11 & $\mathbf{F}$ & $17 \cdot 0$ & $14 \cdot 8$ & $1 \cdot 0$ & $15 \cdot 8$ & $16 \cdot 0$ & $16 \cdot 0$ \\
\hline 12 & $\mathbf{F}$ & $18 \cdot 2$ & $14 \cdot 0$ & 0.8 & $14 \cdot 8$ & $15 \cdot 0$ & $14 \cdot 8$ \\
\hline 13 & $\mathbf{F}$ & $18 \cdot 2$ & $16 \cdot 3$ & $0 \cdot 1$ & $16 \cdot 4$ & $16 \cdot 0$ & $16 \cdot 0$ \\
\hline 14 & $\mathbf{F}$ & $18 \cdot 0$ & $14 \cdot 7$ & $0 \cdot 1$ & $14 \cdot 8$ & $15 \cdot 0$ & $15 \cdot 6$ \\
\hline 15 & $\mathbf{F}$ & $19 \cdot 8$ & $14 \cdot 4$ & 0.7 & $15 \cdot 1$ & $16 \cdot 0$ & $16 \cdot 0$ \\
\hline 16 & F & $14 \cdot 8$ & $13 \cdot 5$ & 0.8 & $14 \cdot 3$ & $13 \cdot 5$ & $13 \cdot 4$ \\
\hline 17 & $\mathbf{M}$ & $19 \cdot 8$ & $16 \cdot 3$ & $1 \cdot 2$ & $17 \cdot 5$ & $17 \cdot 0$ & $17 \cdot 1$ \\
\hline 18 & $\mathbf{F}$ & $17 \cdot 2$ & $12 \cdot 2$ & $3 \cdot 1$ & $15 \cdot 3$ & $15 \cdot 0$ & $15 \cdot 2$ \\
\hline
\end{tabular}




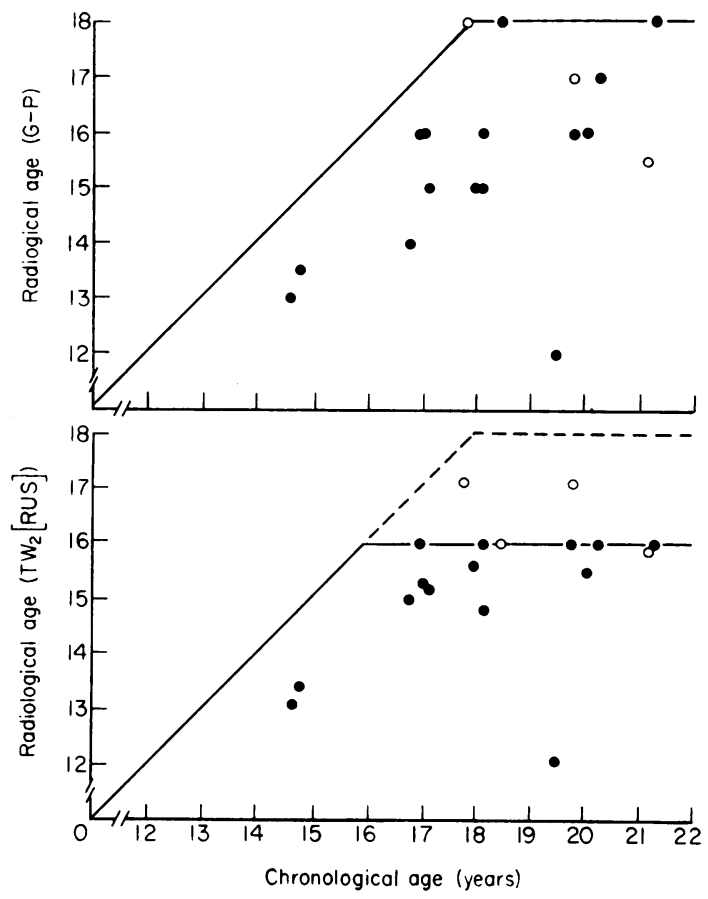

Fig. 1. (a). Comparison of the radiological (G-P) and chronological ages $\bigcirc$ male, female; line represents a theoretical relationship of equality.

(b) Comparison of the radiological (TW2[RUS]) and chronological ages $\bigcirc$ male, female; - females, ....-males, represent a theoretical relationship of equality.

analysed separately using each of these methods. The results were essentially the same in both cases.

Figures $1 \mathrm{a}$ and $1 \mathrm{~b}$ show clearly that there was little or no association between the radiological assessment of age and the chronological age of the patients. For most of the patients it was found that the radiological age (bone maturity) was considerably less than should have been reached at their chronological age. The theoretical relationship to be expected if the radiological age accurately measured the chronological age is illustrated in the figures. It becomes horizontal as soon as the chronological age exceeds the maximum age determinable by the radiological method. Little association was found between the radiological age and the age of onset of anorexia nervosa. For most patients the radiological age was greater than the age of onset.

The relationship between the radiological age and the sum of age of onset of illness plus the period(s) of re-feeding was then examined (Fig. $2 a$ and $b$ ). Up to the maximum age determinable by the radiological method, least squares regression analysis showed a highly significant $(P<0.001)$ linear relationship which did not differ significantly $(P>0.2$
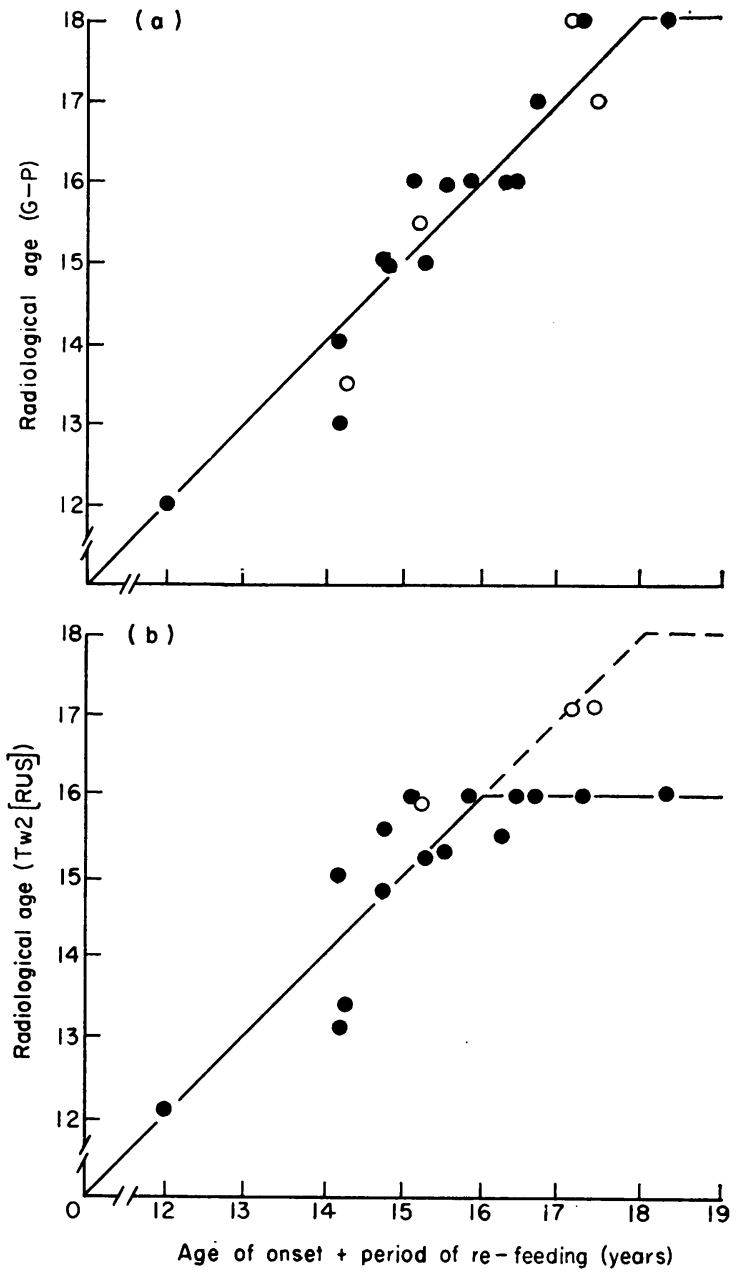

FIG. 2. (a) Comparison of the radiological age (G-P) and the sum of age of onset of anorexia nervosa plus period of re-feeding, $\bigcirc$ male, female; the line represents a theoretical relationship of equality.

(b) Comparison of the radiological age (TW2[RUS]) and the sum of age of onset of anorexia nervosa plus period of re-feeding. $\bigcirc$ male, female; - females; ...--males, represent a theoretical relationship of equality.

G-P method, $P>0.8$ TW2(RUS) method) from a line of slope unity passing through the origin. This indicated a relationship of equality between them.

The standard deviation of the radiological age determinations in this study was estimated from the residual variation about the fitted line and was 6.8 months for the G-P method and 7.9 months for the TW2(RUS) method. The error in assessing age from hand radiographs is well documented (Greulich and Pyle, 1959) for the G-P method and varies with age and sex. The observed error agreed well with this. 
The radiological assessments of age given by the 2 methods in this study were not significantly different $(P>0 \cdot 8)$. Also the standard deviations of the methods did not differ significantly $(P>0 \cdot 5)$.

\section{Discussion}

The finding that patients with early onset anorexia nervosa tend to be shorter than their peers (Crisp, 1969 ) is consonant with the present finding that bony maturation becomes retarded during periods of active anorectic illness. Further, there is a strong suggestion from the data that bony development actually ceases when the weight loss has been sufficient to stop menstruation. Certainly the 4 patients (numbers $3,5,7$ and 8 ) who had persistently starved themselves throughout the course of their illness, showed no radiological evidence of maturing since the onset of their anorexia.

There is a highly significant agreement between the radiological age and sum of the age of onset of the illness plus time spent re-feeding. Adequate renourishment and weight gain seem to rekindle the bone-maturing mechanism. This suggests that bone maturation since the onset of illness is similar in time to the period of re-feeding and thus there is little evidence of accelerated 'catch up' with true chronological age.

It seems possible that bone age, as estimated from radiographs, more accurately reflects a patient's emotional stage of development than does the chronological age; and that the data presented here add to the substantial evidence that the anorectic is biologically and psychologically immature for age (Crisp, 1977). None of the patients in this sample vomited or purged him/herself and his/her serum electrolytes were within normal range. All of them had gone through a substantial period of low calorific intake to achieve a low but stable body weight and, at the time of the study, were in calorific balance. Their energy intake (Crisp, Leatham and Lacey, 1978) was at the lower end of the range ingested by their peers (Lacey et al., 1978) and they were eating adequate amounts of proteins and fats. The essential features demonstrated by the patients in this study were that they were almost totally abstinent of carbohydrate, and were at a low body weight and body fat. Their body weights were below the critical threshold for biological sexuality and consequently they could be expected to have low serum levels of those hormones associated with reproduction, also growth hormone, but high levels of cortisol (Kalucy et al., 1976). Whilst it is generally accepted that skeletal maturity is under endocrine control, the relative hormone balance needed for each stage of bone maturity is unknown. Gonadotrophins are necessary for epiphyseal fusion and bone maturation during the adolescent period that this illness thwarts. Further cortisol levels in doses not much in excess of the normal rate of secretion retards skeletal maturation (Tanner et al., 1975).

Whilst radiological assessment of bone age would contribute to a true prediction of the degree of immaturity, regular monitoring during treatment would seem to be unjustified because body weight is a sufficient guide to the progress of physical development.

Major dieting is endemic and epidemic amongst normal schoolgirls (Huenemann et al., 1966), and manipulation of diet commonplace (Lacey et al., 1978) such that some $10 \%$ of girls diet sufficiently for menstruation to cease (Nylander, 1971). Further, it is known that $1-2 \%$ of adolescent females develop anorexia nervosa (Crisp, Palmer and Kalucy, 1976). It thus appears that many young females go through periods of serious abstention from carbohydrate and a substantial number allow their weight to fall below the critical thresholds mentioned above needed to maintain the hormonal drive for growth in both a biological and emotional sense, perhaps thus interfering significantly with their physical and psychological development.

\section{References}

CRISP, A.H. (1969) Some skeletal measurements in patients with primary anorexia nervosa. Journal of Psychosomatic Research, 13, 125.

CRISP, A.H. (1970) Anorexia nervosa, 'feeding disorder', 'nervous malnutrition' or 'weight phobia'. World Review of Nutrition and Dietetics, 12, 452.

CRISP, A.H. (1977) Some psychobiological aspects of adolescent growth and their relevance for the fat/thin syndrome (anorexia nervosa). International Journal of Obesity, 1, 231.

CrisP, A.H., LeAtham, S. \& LACEy, J.H. (1978) Dietary intake in anorexia nervosa. (In preparation.)

CrisP, A.H., Palmer, R.L. \& Kalucy, R.S. (1976) How common is anorexia nervosa? A prevalence study. British Journal of Psychiatry, 128, 549.

FrisCh, R.E. \& MCARThUR, J.W. (1974) Menstrual cycles fatness as a determinant of minimum weight for height necessary for maintenance or onset. Science. New York, etc., $185,949$.

Greulich, W.W. \& Pyle, S.I. (1959) Radiographic Atlas of Skeletal Development of Hand and Wrist, 2nd edn. Stanford University Press, California.

Huenemann, R.L., Shapiro, L.R., Hampton, M.C. \& Mitchell, B.E. (1966) A longitudinal study of gross body composition and body conformation and their association with food and activity in a teenage population. American Journal of Clinical Nutrition, 18, 325.

Kalucy, R.S., Crisp, A.H., Chard, T., McNeilly, A., Chen, C.N. \& LACEY, J.H. (1976) Nocturnal hormonal profiles in massive obesity, anorexia nervosa and normal females. Journal of Psychosomatic Research, 20, 595.

Lacey, J.H., Chadbund, C., Crisp, A.H. \& Whitehead, J. (1978) 'Dietary patterns in normal schoolgirls'. British Journal of Human Nutrition, 32, 419.

MONEY, J. (1975) Leading address. Second International Sleep Research Congress, Edinburgh. Karger, Basel. 
NyLANDER, I. (1971) The feeling of being fat and dieting in a school population: epidemiologic interview investigation. Acta sociomedica scandinavica, 3, 17.

Palmer, R.L., Crisp, A.H., MacKinnon, P.C.B., Franklin, M., Akando, E.O. \& Bonnar, J. (1974) Gonadatrophin response to L.H./F.S.H.-R.H. during weight gain in patients with anorexia nervosa. Journal of Endocrinology, 63, 32.

Powell, G.F., Hopwood, N.S. \& Barratt, E.S. (1973) Growth hormone studies before and during catch-up growth in a child with emotional deprivation and short stature. Journal of Clinical Endocrinology and Metabolism, 37, 674.

Prader, A., Tanner, J.M. \& von Harnack, G.A. (1963) Catch-up growth following illness or starvation. Journal of Pediatrics, 62, 646.

TANNer, J.M., Whitehouse, R.H. \& Marshall, W.A. (1975) Assessment of Skeletal Maturity and Prediction of Adult Height (T.W.2 Method). Academic Press, London.

Toms, D.A. \& CRISP, A.H. (1972) Weight phobia in an adolescent male with stunted development. Journal of Psychosomatic Research, 16, 289. 